\title{
¿Por qué los estudiantes dejan todo para última hora? Variables implicadas en el aprendizaje autorregulado Why do students leave everything to the last minute? Variables underlying self- regulation learning
}

\author{
Elena Escolano-Pérez, Mª́ngeles Bravo-Álvarez, Ma Luisa Herrero-Nivela, Marian Acero-Ferrero \\ eescola@unizar.es,marian@unizar.es,mherniv@unizar.es, macero@unizar.es \\ Departamento de Psicología y Sociología \\ Universidad de Zaragoza \\ Zaragoza, España
}

\begin{abstract}
Resumen- La procrastinación académica (tendencia irracional a demorar la realización de una tarea académica) afecta a numerosos estudiantes, perjudicando su aprendizaje y rendimiento académico además de su salud física y psicológica. Bajo estos hábitos procrastinadores subyacen deficits en autorregulación, habilidad imprescindible para el aprendizaje autónomo que todo universitario debe desarrollar. A su vez, estos déficits autorregulatorios se deben a niveles inadecuados en distintas variables cognitivas, metacognitivas y motivacionales. Los objetivos de este trabajo son conocer el nivel de procrastinación que presenta el alumnado universitario de nuevo ingreso, así como determinar qué variables cognitivas, metacognitivas y motivacionales pueden predecirlo. Además, se pretende conocer si existen diferencias tanto en el nivel de procrastinación como en las variables predictoras del mismo en función del Grado que cursan los estudiantes que componen la muestra: $\mathrm{N}=303$ estudiantes de los Grados en Magisterio en Educación Infantil $\left(\mathrm{n}_{1}=113\right)$ y Magisterio en Educación Primaria $\left(n_{2}=190\right)$. Los resultados indican que la muestra presenta un nivel de procrastinación medio, no existiendo diferencias en función del Grado que cursan los participantes. Sin embargo, las variables predictoras del mismo sí varían entre los estudiantes de cada Grado. Se discuten las implicaciones educativas de estos resultados en cuanto a su utilidad para diseñar actividades preventivas en cada Grado.
\end{abstract}

Palabras clave: Procrastinación, autorregulación, aprendizaje autónomo, cognición, metacognición, motivación, alumnado universitario de nuevo ingreso.

Abstract- Academic procrastination (irrational tendency to delay of an academic task) affects many students, impairing their learning and academic performance in addition to their physical and psychological health. The reason behind the problem of procrastination are deficits in self-regulation, an essential skill for the autonomous learning that every university student must develop. These self-regulatory deficits are in turn caused by inadequate levels in different cognitive, metacognitive and motivational variables. Aims of this study are to know the level of procrastination in beginner university students and to determine which cognitive, metacognitive and motivational variables can predict their level of procrastination. In addition, this study pretends to know whether there are differences in the level of procrastination of students and in their predictor variables depending the Degree of the students that compose the sample: $\mathrm{N}=303$ students of the Early Childhood Education Degree $\left(n_{1}=113\right)$ and of the
Primary Education Degree $\left(\mathrm{n}_{2}=190\right)$. Results indicate that the sample shows a medium level of procrastination and there are no differences between the two groups of students depending on their Degree. However, the predictor variables of the procrastination vary among students of the two Degree. The results are discussed in relation to their usefulness in deriving intervention guidelines within the each degree program.

Keywords: Procrastination, self-regulation, autonomous learning, cognition, metacognition, motivation, beginner university student.

\section{INTRODUCCIÓN}

La procrastinación académica, es decir, la tendencia irracional a demorar el inicio y/o realización de una tarea académica (Senecal, Julien \& Guay, 2003) afecta a numerosos estudiantes, perjudicando ya no solo su desempeño y rendimiento académico sino incluso su salud física y psicológica (Özer \& Saçkes, 2011). En el nivel universitario, la situación se agrava (Saracaloglu \& Gökdas, 2016) dado que el aprendizaje autónomo del estudiante, y por tanto su capacidad e implicación para asumir la responsabilidad y organización de su trabajo y estudio, adquiere un rol esencial en su formación. De hecho, numerosos autores manifiestan que la procrastinación tiene como procesos subyacentes déficits autorregulatorios, siendo la autorregulación una habilidad imprescindible para el aprendizaje autónomo que todo universitario debe adquirir.

Numerosos estudios han tratado de determinar los factores que llevan al alumnado a procrastinar, afirmando que se trata tanto de variables personales (de tipo cognitivo, metacognitivo y motivacional, además de rasgos de personalidad) como variables sociodemográficas y factores del contexto psicosocial (Boysan \& Kiral, 2017). Sin embargo, no existe consenso total al tratar de identificar cada una de ellas.

Este trabajo analiza el nivel de procrastinación que presenta el alumnado de primer curso de los Grados en Magisterio en Educación Infantil y Magisterio en Educación Primaria, así como algunas de las variables personales individuales que están contribuyendo a ello. Se presenta un modelo predictivo de la procrastinación de los estudiantes a partir del análisis de distintas variables cognitivas, metacognitivas y motivacionales 
consideradas por diversos autores variables predictoras importantes de los hábitos procrastinadores académicos. Nos centramos en este tipo de variables (cognitivas, metacognitivas y motivacionales) ya que son variables posibles de modificar a través de intervención en el contexto universitario, frente a otras variables (por ejemplo, el nivel socioeconómico del alumnado) que, aun pudiendo afectar el nivel de procrastinación del alumnado, queda fuera de nuestras posibilidades intervenir sobre ellas de cara a eliminar los hábitos procrastinadores del alumnado.

Los resultados de nuestro trabajo arrojan modelos predictivos de la procrastinación diferentes para cada grupo de estudiantes (y ello a pesar de ser todos alumnos de los Grados en Magisterio), indicando que las variables que están potenciando hábitos procrastinadores en los estudiantes varían en función del Grado en Magisterio que estos cursan (Educación Infantil o Educación Primaria). Estos resultados son de gran relevancia en cuanto que, a partir de ellos, se está en disposición de diseñar e implementar actividades que, ajustadas a las características y necesidades de cada grupo de estudiantes, permitan disminuir e incluso erradicar sus hábitos procrastinadores, favoreciendo así su aprendizaje de calidad y su desempeño académico eficiente.

En definitiva, este trabajo nos permite conocer mejor el fenómeno de la procrastinación en nuestro contexto académico y su relación con otras variables personales, posibilitando el diseño de una intervención dirigida a su erradicación y por tanto, potenciando resultados más favorables para quienes la padecen. En última instancia, se trata de reducir el impacto no solo personal sino también institucional y social de este problema que constituye la procrastinación académica.

\section{CONTEXTO}

\section{A. Necesidad de realización del estudio}

Somos muchos los profesores que observamos que gran cantidad de nuestros estudiantes dejan todo para el último momento. Es lo que comúnmente se denomina dilación o postergación; y en la literatura, "procrastinación”. Muchos autores consideran que a la base de las conductas procrastinadoras se encuentra un déficit en autorregulación (Balkis \& Duru, 2016; Motie, Heidari, \& Sadeghi, 2012). Ello podría justificar el incremento de la prevalencia de la procrastinación en el nivel universitario, frente a niveles educativos previos, pues es en el Espacio Europeo de Educación Superior donde adquiere un papel esencial el aprendizaje autorregulado -también denominado aprendizaje autónomo, aprendizaje autodirigido, aprendizaje independiente o autoaprendizaje (Suárez Riveiro y Fernández Suárez, 2016)-.

En el aprendizaje autónomo, el estudiante es sujeto activo de su aprendizaje y por tanto, responsable de la organización de su trabajo y de la adquisición de las diferentes competencias según su propio ritmo. Ello le exige establecer sus propios objetivos y tomar decisiones sobre la planificación, realización y evaluación de la experiencia de aprendizaje (Lobato, 2009). En definitiva, el estudiante debe controlar su proceso personal de aprendizaje logrando monitorizar, regular y controlar su cognición, motivación y comportamiento. La iniciativa personal y la perseverancia en la tarea son elementos esenciales para un aprendizaje autónomo exitoso (Rosário et al., 2010). Actualmente, y en un futuro inmediato, ser autónomo constituye una competencia imprescindible para sobrevivir y progresar en una sociedad configurada por numerosos, rápidos e imprescindibles cambios. Consecuentemente, el aprendizaje autónomo constituye un concepto clave en la Educación Superior: formar a los estudiantes para la autonomía en su aprendizaje es una de las principales finalidades de la educación formal, especialmente en la etapa universitaria (Lobato, 2009).

El aprendizaje autónomo en estudiantes universitarios parece estar constituido por tres importantes aspectos (Pintrich \& Groot, 1990): 1) estrategias cognitivas o procedimientos intencionales que permiten al estudiante tomar las decisiones oportunas de cara a mejorar su estudio y rendimiento (Lobato, 2009). Entre ellas se encuentra el control del tiempo (Roces, Tourón y González, 1995); 2) estrategias metacognitivas: son herramientas que ayudan al sujeto a tomar conciencia de su proceso de aprendizaje, haciéndolo capaz de autorregular dicho aprendizaje. Así, el uso de unas adecuadas estrategias metacognitivas permiten al estudiante planificar, autocontrolar y autoevaluar el empleo eficaz de los propios recursos cognitivos en función de las características de la tarea a la que se enfrenta. Por tanto, utilizar unas adecuadas estrategias metacognitivas implica tener conocimiento de la tarea a realizar y de sí mismo, entre otras cuestiones (Allueva, 2002); y 3) estrategias de apoyo: se refieren al control que ejerce el estudiante sobre una serie de variables no intelectuales que influyen en su implicación en la tarea, es decir, implican la promoción de condiciones que faciliten afectivamente el estudio. Entre ellas se incluye la disposición motivacional, enlazada a su vez con el interés e importancia otorgados a la tarea a realizar, la percepción de capacidad para afrontarla exitosamente, la creencia y sensación de dominio sobre la tarea y los resultados esperados (Lobato, 2009).

$\mathrm{Si}$ estas estrategias cognitivas, metacognitivas y motivacionales se encuentran implicadas en el aprendizaje autónomo o autorregulado - y la procrastinación constituye un déficit de autorregulación-, déficits en dichas estrategias potenciarán hábitos procrastinadores en los estudiantes, con importantes consecuencias asociadas. Estas consecuencias no se limitan únicamente al ámbito académico del estudiante (menor calidad y cantidad de aprendizaje y rendimiento académico) sino que afectan incluso a su bienestar y salud física y psicológica (se realiza menos ejercicio físico; no se tienen hábitos alimentarios saludables; mayores niveles de estrés, ansiedad y depresión; problemas en el manejo de las relaciones sociales, etc.). (Özer \& Saçkes, 2011). Es por todo ello que consideramos necesario abordar el problema de la procrastinación en nuestro alumnado.

Analizar las variables cognitivas, motivacionales y metacognitivas en nuestros alumnado y su contribución a sus hábitos procrastinadores aportará información relevante para el diseño de actividades de intervención dirigidas a la erradicación y prevención en el futuro de este problema que constituye la procrastinación. Prevenir la procrastinación en los estudiantes siempre es necesario, pero más aún cuando estos se encuentran iniciando una nueva etapa educativa con características y exigencias muy diferentes a las anteriores (Motie et al., 2012), tal y como es el caso de los participantes en este estudio. Hay evidencia de que la procrastinación, en caso de no intervenir, es una conducta particularmente estable que se mantiene desde el inicio hasta el final del periodo académico (Rice, Richardson, \& Clark, 2012), de modo que los hábitos adquiridos durante el primer año universitario 
tienen consecuencias perdurables en la formación y vida del alumnado. Una de las repercusiones más drásticas de la procrastinación en la formación del alumnado es el abandono de sus estudios. La literatura más actual incluye la procrastinación como una de las variables que más contribuyen al fracaso y abandono universitario (Garzón Umerenkova y Gil Flores, 2017). Ambos fenómenos (procrastinación y abandono universitario) muestran su mayor prevalencia en el primer curso universitario (Esteban García, Bernardo Gutiérrez y Rodríguez-Muñiz, 2016). En nuestra Universidad (Universidad de Zaragoza), la tasa de abandono en el primer año es del 18,5\% (Ministerio de Educación, Cultura y Deporte, 2016), y si bien se encuentra por debajo de la media nacional, los costos económicos y sociales que conlleva son enormes. Asimismo, hay que considerar que de no erradicar la procrastinación académica, esta tiende a generalizarse a otros ámbitos de la vida como el laboral, familiar o social (Mann, 2016). Por tanto, queda patente que el fenómeno de la procrastinación constituye un problema que no se circunscribe a la esfera de lo personal y académico de aquel que la padece, sino que alcanza lo familiar, económico y social), por lo que bien queda justificado la necesidad de su abordaje.

\section{B. Objetivos}

En relación con todo lo expuesto anteriormente, los objetivos de nuestro trabajo fueron los siguientes:

\section{* Objetivos generales}

1- Conocer el nivel de Procrastinación del alumnado universitario de nuevo ingreso que forma parte de la muestra de estudio.

2- Determinar un modelo predictivo de la Procrastinación en el alumnado universitario de nuevo ingreso que conforma la muestra de estudio a partir de variables personales cognitivas, metacognitivas y motivacionales evaluadas en estos.

\section{* Objetivos específicos}

1- Conocer el nivel de Procrastinación del alumnado de nuevo ingreso de cada uno de los Grados analizados (Magisterio en Educación Infantil y Magisterio en Educación Primaria) y la existencia de posibles diferencias entre ellos.

2- Determinar un modelo predictivo de la Procrastinación del alumnado de nuevo ingreso en cada Grado (Magisterio en Educación Infantil y Magisterio en Educación Primaria) a partir de las variables cognitivas, metacognitivas y motivacionales evaluadas en los mismos, así como analizar posibles semejanzas y diferencias entre ambos modelos.

\section{Contexto y público objetivo}

La muestra de estudio estuvo formada por 303 estudiantes de primer curso de los Grados en Magisterio en Educación Infantil y Educación Primaria de la Facultad de Educación de la Universidad de Zaragoza. En concreto, 113 estudiantes pertenecían al Grado en Magisterio en Educación Infantil y 190 al Grado en Magisterio en Educación Primaria. La participación en el estudio fue voluntaria. A todos los participantes se les ofreció la posibilidad de recibir un informe individualizado con sus resultados personales así como la participación en un taller destinado al conocimiento y reflexión sobre las variables que afectan a sus hábitos procrastinadores y la puesta en marcha de estrategias y recomendaciones para la mejora de estos.

\section{DESCRIPCIÓN}

\section{A. Metodología}

Para la realización de este estudio se hizo uso de la metodología selectiva. En concreto, se trata de un estudio "ex post facto”, descriptivo y predictivo.

\section{B. Instrumentos}

Los instrumentos utilizados para la recogida de datos fueron los siguientes:

a) Para la recogida de los datos referidos a la variable Procrastinación se utilizó la Escala de Demora Académica (EDA; Clariana y Martín, 2008).

b) Para la recogida de los datos referidos a las variables cognitivas (Gestión del tiempo), se aplicó la versión española del Time Management Behavior Questionnaire (Macan, 1994, 1996), realizada por García-Ros y Pérez-González (2012) para estudiantes universitarios españoles. Consta de cuatro factores o subescalas independientes denominadas: 1.Establecer objetivos y prioridades; 2.- Herramientas para la gestión del tiempo; 3.- Control percibido del tiempo; 4.Preferencia por la desorganización.

c) Para la evaluación de las variables metacognitivas se utilizaron las correspondientes subescalas del Cuestionario CEVEAPEU (Gargallo, Suárez-Rodríguez y Pérez-Pérez, 2009), denominadas de la misma manera que las propias variables evaluadas: Conocimiento de objetivos y criterios de evaluación; Planificación; Autoevaluación; Control/autorregulación.

d) Para la evaluación de las variables motivacionales se utilizó nuevamente el Cuestionario CEVEAPEU (Gargallo et al., 2009), en este caso sus subescalas denominadas de la misma manera que las propias variables motivacionales evaluadas: Motivación intrínseca; Motivación Extrínseca; Valor de la tarea; Atribuciones internas; Atribuciones externas; Autoeficacia y expectativas; Concepción de la inteligencia como modificable.

Todos los instrumentos utilizados para la evaluación de las variables objeto de interés son escalas tipo Likert con buenas propiedades psicométricas para su uso con estudiantes universitarios españoles.

Para el análisis de datos se usó el software estadístico SPSS v. 19.0 .

\section{Procedimiento}

En cada grupo natural de estudiantes (dos grupos de Magisterio en Educación Infantil y cuatro grupos de Magisterio en Educación Primaria), en una de sus clases habituales se dedicaron los 15-20 minutos últimos a la cumplimentación de los instrumentos por parte del alumnado. Cada estudiante debió contestar de manera individual a cada uno de los cuestionarios.

La participación en el estudio fue voluntaria. Se señaló que aquel que lo deseara podía indicar su nombre con el fin de poder recibir posteriormente un informe personalizado con sus resultados. Asimismo, se invitó a todos los estudiantes a la 
participación en un taller dirigido al conocimiento y reflexión sobre las variables que afectan a sus hábitos procrastinadores y la puesta en marcha de estrategias y recomendaciones para la mejora de estos.

Una vez vaciados los datos individuales de cada participante, se procedió a su análisis estadístico para responder a los objetivos de este estudio. Los análisis realizados variaron en función de estos: análisis descriptivos de tendencia central y variabilidad para conocer el nivel de Procrastinación del alumnado (objetivo general 1 y objetivo específico 1); estadístico t para la comparación de medias de dos muestras independientes (objetivo específico 1) y análisis de regresión múltiple por pasos sucesivos para determinar los modelos predictivos de la Procrastinación a partir de las variables personales del alumnado (objetivo general 2 y objetivo específico 2).

\section{RESUltados}

\section{A. Resultados referidos a los objetivos generales}

La muestra presenta una Procrastinación media de 35.17 puntos $(\mathrm{M}=35.17$; $\mathrm{DT}=10.007)$, lo que constituye un nivel medio.

Se ha obtenido un modelo predictivo de la Procrastinación de este alumnado que resulta estadísticamente significativo $(\mathrm{F}=44.579 ; \mathrm{p}=.000)$ y que explica casi la mitad de la varianza de la Procrastinación en la muestra $\left(\mathrm{R}^{2}\right.$ ajustado $\left.=.464\right)$. De acuerdo con Cohen (1988), al ser este valor de $\mathrm{R}^{2}$ superior a .26 indica un tamaño de efecto grande, es decir, las variables incorporadas en el modelo tienen un efecto sustantivo sobre la Procrastinación.

Estas variables que conforman este modelo y que por tanto actúan como predictores significativos de la Procrastinación son, de más a menos importantes de acuerdo al valor de su coeficiente estandarizado $\beta$, las siguientes: Control percibido del tiempo $(\beta=-.327)$; Planificación $(\beta=-.221)$; Establecer objetivos y prioridades $(\beta=-.191)$; Preferencia por la desorganización $(\beta=.133)$; Control/autorregulación $(\beta=-.130)$ y Motivación intrínseca $(\beta=-.96)$. Todas las variables salvo Preferencia por la desorganización inciden de manera negativa en la Procrastinación, de modo que al descender las puntuaciones en dichas variables aumenta la Procrastinación. En cambio, Preferencia por la desorganización incide positivamente en la Procrastinación; es decir, al aumentar la Preferencia por la desorganización aumenta también la Procrastinación. El modelo predictivo formado por estas variables es:

Procrastinación $=91.302-.707$ Control percibido del tiempo - .283 Establecer objetivos y prioridades - 1.079 Planificación - .456 Control/autorregulación + .455 Preferencia por la desorganización - .583 Motivación intrínseca.

En resumen, algunas variables cognitivas referidas a la gestión del tiempo (Control percibido del tiempo; Establecer objetivos y prioridades y Preferencia por la desorganización) además de otras variables metacognitivas (Planificación y Control/autorregulación) y la variable motivacional de Motivación intrínseca explican casi en un 50\% la varianza de las puntuaciones obtenidas en Procrastinación por este alumnado, actuando como predictores significativos de la misma.

\section{B. Resultados referidos a los objetivos específicos}

Los estudiantes de Magisterio en Educación Infantil, de media, presentan un nivel de Procrastinación bajo ( $M=34.82$; $\mathrm{DT}=.772$ ) mientras que aquellos de magisterio en Educación Primaria muestra una nivel de Procrastinación medio $(\mathrm{M}=35.37 ; \mathrm{DT}=$.836). Sin embargo, estadísticamente, no existen diferencias significativas entre el nivel de Procrastinación de ambos grupos de estudiantes $(\mathrm{t}=.479 ; \mathrm{p}=$ .632; IC95\%[-1.694, 2.785]).

En cuanto al modelo predictivo de la Procrastinación del alumnado de Magisterio en Educación Infantil, se ha obtenido un modelo estadísticamente significativo $(\mathrm{F}=28.775 ; \mathrm{p}=.000)$ que con tan solo tres variables predictoras (Establecer objetivos y prioridades; Control percibido del tiempo y Planificación) explica casi un $43 \%$ de la varianza de la Procrastinación de estos alumnos $\left(\mathrm{R}^{2}\right.$ ajustado= .427$)$, indicando además un tamaño de efecto grande (Cohen, 1988). La variable que más peso tiene en el modelo es Control percibido del tiempo $(\beta=-.331)$; seguida de Establecer objetivos y prioridades $(\beta=-.271)$ y por último, Planificación $(\beta=-.257)$. Todas ellas influyen de manera negativa a la Procrastinación, de manera que al descender sus puntuaciones aumenta la Procrastinación del alumnado de Magisterio en Educación Infantil.

El modelo predictivo de la Procrastinación de este alumnado del Grado en Magisterio en Educación Infantil es:

Procrastinación Grado Magisterio Educación Infantil = 82.834 - .357 Establecer objetivos y prioridades - .707 Control percibido del tiempo - 1.119 Planificación

Llama la atención que en los estudiantes de Magisterio en Educación Infantil las variables motivacionales no tienen ningún efecto sobre la Procrastinación de los estudiantes, pues solo han resultado predictores significativos de la misma dos variables cognitivas y una metacognitiva.

En lo referido al alumnado del Grado en Magisterio en Educación Primaria, sus hábitos procrastinadores pueden predecirse a partir de un modelo estadísticamente significativo $(\mathrm{F}=30.583 ; \mathrm{p}=.000)$ que explica casi la mitad de la varianza de su Procrastinación $\left(\mathrm{R}^{2}\right.$ ajustado $\left.=.484\right)$ con un tamaño del efecto grande (Cohen, 1988). Este modelo se compone de seis variables predictoras estadísticamente significativas. Su peso en el modelo, de mayor a menor y de acuerdo a su coeficiente estandarizado, es: Control percibido del tiempo $(\beta=-.378)$; seguida de Control/autorregulación y Herramientas para la gestión del tiempo, cuyos coeficientes estandarizados presentan el mismo valor $(\beta=-.187)$; Herramientas para la gestión del tiempo $(\beta=-.187)$; Planificación $(\beta=-.176)$; Atribución externa $(\beta=.138)$ y Motivación intrínseca $(\beta=-$ .131). La Atribución externa es la única variable que incide en sentido positivo en la Procrastinación, de modo que un incremento en la misma conlleva un incremento en la Procrastinación. En cambio, descensos en las puntuaciones del resto de variables predictoras producen asimismo un incremento en la Procrastinación.

El modelo predictivo de la Procrastinación del alumnado del Grado en Magisterio en Educación Primaria es:

Procrastinación Grado Magisterio Educación Primaria = 96.671 - .823 Control percibido del tiempo - .661 Control/autorregulación - .275 Herramientas para la gestión 
del tiempo - .911 Planificación + .939 Atribución externa .850 Motivación intrínseca.

En este caso, y comparativamente al modelo predictor de la Procrastinación del alumnado de Magisterio en Educación Infantil, se aprecia que aunque el modelo explica un 5.7\% más de la varianza de la Procrastinación, está formado por un mayor número de variables personales y además tanto de tipo cognitivo como metacognitivo y motivacional. Solo existen dos variables que actúan como predictores significativos de la Procrastinación en ambos grupos de estudiantes: la variable cognitiva Control percibido del tiempo y la variable metacognitiva Planificación.

En conclusión, los modelos predictivos obtenidos explican cerca del 50\% de la varianza de la Procrastinación, tanto de la muestra en conjunto como de los estudiantes de cada Grado en Magisterio. Sin embargo, en el caso de los estudiantes de Magisterio en Educación Infantil su Procrastinación puede predecirse a partir de tres únicas variables (de tipo cognitivo y metacognitivo) mientras que en el caso de los estudiantes de Magisterio en Educación Primaria son el doble de variables las que actúan como predictoras de su Procrastinación, siendo además tanto de tipo cognitivo y metacognitivo como motivacionales. Por lo tanto, la Procrastinación parece ser un fenómeno más complejo de comprender y predecir en los estudiantes de Magisterio en Educación Primaria que en los de Magisterio en Educación Infantil, dada la mayor cantidad y variedad de procesos que subyacen a la misma.

\section{CONCLUSIONES}

Este trabajo supone un mayor conocimiento del problema que constituye la procrastinación en el alumnado de nuevo ingreso en los Grados en Magisterio. En este sentido, se ha podido conocer que a pesar de que el número y tipología de variables que ejercen un efecto sustantivo sobre la Procrastinación de los estudiantes de Magisterio en Educación Primaria es superior al de variables que hacen lo propio en los estudiantes de Magisterio en Educación Infantil, en ambos casos la Planificación y distintas cuestiones referidas a la Gestión del tiempo resultan tener una incidencia negativa sobre la Procrastinación del alumnado. De este modo, dificultades en estas variables contribuyen al aumento de la Procrastinación. Estas dificultades podrían deberse, a su vez, a la falta de oportunidades y experiencias previas de aprendizaje que requieren la puesta en marcha de dichas habilidades por parte del alumnado. En los niveles educativos previos a la universidad es el profesorado quien establece y determina los objetivos, tareas y tiempo para su ejecución, constituyendo además frecuentemente tareas bien definidas, estructuradas y a corto plazo, con supervisión y corrección individualizada por parte del profesorado y con consecuencias inmediatas (especialmente en caso de que no hayan sido realizadas). Así, los estudiantes, en el mejor de los casos, se limitan a ejecutar la planificación establecida por el profesorado, de modo que alcanzan los estudios universitarios sin hacer uso de una planificación y gestión del tiempo "personal ni propia". Además, la mayor libertad personal y académica del estudiante universitario junto al cada vez mayor número de distracciones existentes en las sociedades avanzadas (siendo además estas distracciones de acceso inmediato y continuo: redes sociales, telefonía móvil, etc.), hacen que el estudio y trabajo sea un hábito difícil de instaurar en el alumnado.
Sin embargo, la Planificación y Gestión del tiempo son variables modificables y por tanto, pueden ser objeto de intervención. En este sentido, los resultados de nuestro estudio permiten diseñar acciones preventivas y optimizadoras que bien podrían formar parte del Plan de Orientación Universitaria de la Facultad de Educación de la Universidad de Zaragoza, tanto en su fase I dirigida a estudiantes de nuevo ingreso como en su fase II dirigida a alumnado de los restantes cursos (Allueva, Zulaica y Abadía, 2016). Así, se estaría en disposición de hacer un seguimiento para asegurar que la eficacia de dichas actuaciones persiste a lo largo de los años.

En este sentido, una estrategia que podría prevenir y mejorar los hábitos procrastinadores de los estudiantes sería llevar a cabo un taller en el que a través de una metodología activa y participativa basada principalmente en el análisis y discusión de lecturas, reflexión personal y práctica dirigida se abordaran los siguientes aspectos referidos a Procrastinación, Planificación y Gestión del tiempo: 1.- Autoconocimiento del empleo del tiempo: análisis de las actividades (académicas y no) que se realizan semanalmente, su distribución y dedicación temporal. 2.- A partir del análisis previo, reconocimiento personal de hábitos procrastinadores e identificación de los aspectos que llevan a ello. 3.Establecimiento de objetivos realistas a corto, medio y largo plazo, distinguiendo asimismo la relevancia y urgencia de cada uno de ellos. 4.- Planificación semanal: anotar las cosas a hacer (incluyendo asimismo actividades de ocio y descanso y otras responsabilidades además de las académicas), cuándo se realizarán y el tiempo previsto destinado a cada una de ellas, teniendo en cuenta además su dificultad y los momentos personales en los que se es más productivo. 5.- Planificación de las sesiones de estudio: establecer prioridades (entre las distintas asignaturas y dentro de cada una de ellas); planificar especialmente las asignaturas y tareas que resultan difíciles y/o de menor agrado; planificar el entorno de estudio; establecer pequeñas metas de estudio específicas; establecer pequeños descansos regularmente (Pérez-González y García-Ros, 2008). Experiencias desarrolladas en esta línea -incluso en tan solo cuatro sesiones (a razón de una por semana)- han arrojado resultados positivos (Nordby, Wang, Dahl y Svartdal, 2016; Rosário et al., 2010).

La sostenibilidad de este trabajo es alta, dado que se han utilizado instrumentos de medida válidos y fiables de carácter gratuito (extraídos de la literatura científica consultada) y de aplicación colectiva. Además, la administración de los instrumentos de medida no exige personal especializado, siendo el propio profesorado quien la realiza.

La muestra de este estudio estaba compuesta por estudiantes de los Grados en Magisterio en Educación Infantil y Magisterio en Educación Primaria, pero este trabajo es transferible a estudiantes de cualquier Grado y cualquier curso, pues la procrastinación puede afectar a cualquier estudiante, independientemente de los estudios que se encuentre realizando y el curso en el que se halle. Conocer qué variables son las que en cada Grado están afectando a los hábitos procrastinadores del alumnado permitirá el diseño de acciones preventivas exitosas. La procrastinación es modificable, y cuanto antes se elimine generando conductas alternativas y adecuadas para el estudio, antes mejorará el desempeño y rendimiento académico de los estudiantes. Ello contribuirá a la formación de profesionales competentes, independientemente de cuál sea su campo de especialización. 


\section{AGRADECIMIENTOS}

Este trabajo forma parte del Proyecto de Innovación Docente (código PIIDUZ_15_152) subvencionado por el Vicerrectorado de Política Académica de la Universidad de Zaragoza.

\section{REFERENCIAS}

Allueva, P. (2002). Desarrollo de Habilidades Metacognitivas: Programa de Intervención. Zaragoza: Consejería de Educación y Ciencia. Diputación General de Aragón.

Allueva, P., Zulaica, F. y Abadía, A. R. (2016). Plan de Orientación Universitaria de la Universidad de Zaragoza. Integración de los estudiantes en la Universidad: Binomio Tutor-Mentor. En J. L. Castejón (Coord.), Psicología y Educación: Presente y Futuro (pp. 22732281). Madrid: Asociación Científica de Psicología y Educación.

Balkis, M., \& Duru, E. (2016). Procrastination, self-regulation failure, academic life satisfaction, and affective wellbeing: underregulation or misregulation form. European Journal of Psychology of Education, 31(3), 439-459.

Boysan, M., \& Kiral, E. (2017). Associations between procrastination, personality, perfectionism, self-esteem and locus of control. British Journal of Guidance \& Counselling, 45(3), 284-296. doi:10.1080/03069885.2016.1213374

Clariana, M. y Martín, M. (2008). Escala de Demora Académica. Revista de Psicología General y Aplicada, 61(1), 37-51.

Esteban García, M., Bernardo Gutiérrez, A. B. y RodríguezMuñiz, L. J. (2016). Permanencia en la universidad: la importancia de un buen comienzo. Aula Abierta, 44(1), 1-6. doi:10.1016/j.aula.2015.04.001

García-Ros, R., \& Pérez-González, F. (2012). Spanish Version of the Time Management Behavior Questionnaire for University Students. The Spanish Journal of Psychology, 15(3), 1485-1494.

Gargallo, B., Suárez-Rodríguez, J. M. y Pérez-Pérez, C. (2009). El cuestionario CEVEAPEU. Un instrumento para la evaluación de las estrategias de aprendizaje de los estudiantes universitarios. RELIEVE, 15(2), 1-31.

Garzón Umerenkova, A. y Gil Flores, J. (2017). El papel de la procrastinación académica como factor de la deserción universitaria. Revista Complutense de Educación, 28(1), 307-324. doi:10.5209/rev_RCED.2017.v28.n1.49682

Lobato, C. (2009). Estudio y trabajo autónomos del estudiante. En M. de Miguel (Coord.), Metodologías de enseñanza y aprendizaje para el desarrollo de competencias. Orientaciones para el profesorado universitario ante el Espacio Europeo de Educación Superior (pp. 191-223). Madrid: Alianza Editorial.

Macan, T. H. (1994). Time Management: Test of a Process Model. Journal of Applied Psychology, 79(3), 381-391.

Macan, T. H. (1996). Time-management training: Effects on time behaviors, attitudes, and job performance. The Journal of Psychology, 130, 229-236.
Mann, L. (2016). Procrastination Revisited: A Commentary. Australian Psychologist, 51(1), 47-51. doi: 10.1111/ap.12208

Ministerio de Educación, Cultura y Deporte (2016). Datos y cifras del sistema universitario español. Curso 20152016. Madrid: Secretaría General Técnica. Subdirección General de Documentación y Publicaciones.

Motie, H., Heidari, M., \& Sadeghi, M. A. (2012). Predicting Academic Procrastination during Self-Regulated Learning in Iranian first Grade High School Students. Procedia - Social and Behavioral Sciences, 69, 22992308. https://doi.org/10.1016/j.sbspro.2013.02.023

Nordby, K., Wang, C. E. A., Dahl, T. I., \& Svartdal, F. (2016). Intervention to reduce procrastination in first-year students: Preliminary results from a Norwegian study. Scandinavian Psychologist, 3, e10. https://doi.org/10.15714/scandpsychol.3.e10

Özer, B. U., \& Saçkes, M. (2011). Effects of academic procrastination on college students' life satisfaction. Procedia Social and Behavioral Sciences, 12, 512-519. doi:10.1016/j.sbspro.2011.02.063

Pérez-González, F. y García-Ros, R. (2008). Gestión del tiempo para estudiantes. Pon el tiempo en su sitio. Valencia: Universitat de València.

Pintrich, P. R., \& De Groot, E. V. (1990). Motivational and Self-Regulated Learning Components of Classroom Academic Performance. Journal of Educational Psychology, 82(1), 33-40.

Rice, K. G., Richardson, C. M., \& Clark, D. (2012). Perfectionism, procrastination, and psychological distress. Journal of Counseling Psychology, 59(2), 288302. doi: 10.1037/a0026643. doi: 10.1037/a0026643

Roces, C., Tourón, J. y González, M. C. (1995). Validación preliminar del CEAM II. (Cuestionario de Estrategias de Aprendizaje y Motivación II). Psicológica, 16(3),347366.

Rosário, P., Núñez, J. C., González-Pienda, J., Valle, A., Trigo, L. y Guimarães, C. (2010). Enhancing selfregulation and approaches in first year college students: A narrative-based program assessed in the Iberian Peninsula. European Journal of Psychology of Education, 25, 411-428.

Saracaloglu, A. S., \& Gökdas, I. (2016). Variables that predict academic procrastination behavior in prospective primary school teachers. Journal of Educational Sciences Research, 6(1), 43-61. doi: 10.12973/jesr.2016.61.3

Senecal, C., Julien, E., \& Guay, A. (2003). Role conflict and academic procrastination: A self-determination perspective. European Journal of Social Psychology, 33, 135-145.doi: 10.1002/ejsp.144

Suárez Riveiro, J. M. y Fernández Suárez, A. P. (2016). El Aprendizaje Autorregulado: Variables Estratégicas, Motivacionales, Evaluación e Intervención. Madrid: Universidad Nacional de Educación a Distancia. 